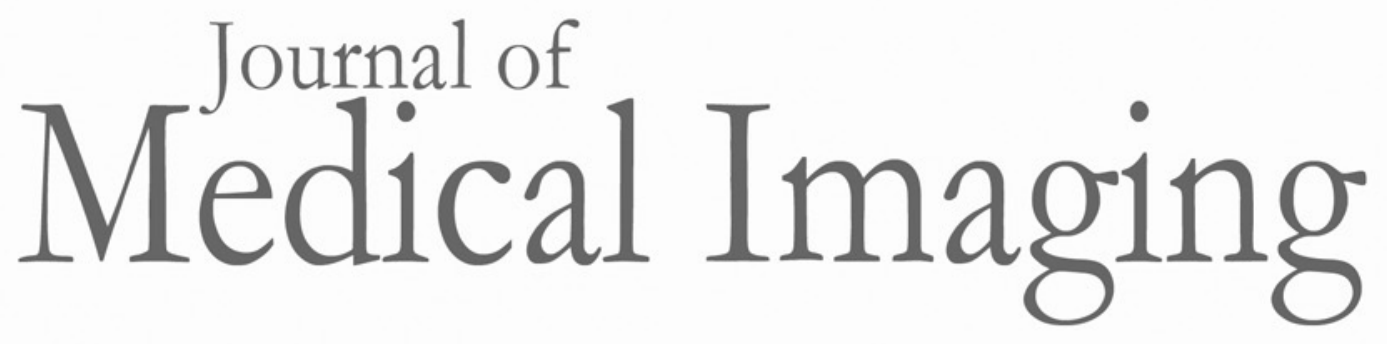

Medicallmaging.SPIEDigitalLibrary.org

\title{
Quantitative sparse array vascular elastography: the impact of tissue attenuation and modulus contrast on performance
}

Steven Huntzicker

Rohit Nayak

Marvin M. Doyley

\section{SPIE.}




\title{
Quantitative sparse array vascular elastography: the impact of tissue attenuation and modulus contrast on performance
}

\author{
Steven Huntzicker, ${ }^{a}$ Rohit Nayak, ${ }^{a}$ and Marvin M. Doyley, ${ }^{\mathrm{a}, \mathrm{b}, *}$ \\ a University of Rochester, Hajim School of Engineering and Applied Sciences, Department of Electrical and Computer Engineering, Rochester, \\ New York 14627 \\ bUniversity of Rochester, Hajim School of Engineering and Applied Sciences, Department of Biomedical Engineering, Rochester, New York 14627
}

\begin{abstract}
Quantitative sparse array vascular elastography visualizes the shear modulus distribution within vascular tissues, information that clinicans could use to reduce the number of strokes each year. However, the low transmit power sparse array (SA) imaging could hamper the clinical usefulness of the resulting elastograms. In this study, we evaluated the performance of modulus elastograms recovered from simulated and physical vessel phantoms with varying attenuation coefficients $\left(0.6,1.5\right.$, and $\left.3.5 \mathrm{~cm}^{-1}\right)$ and modulus contrasts $(-12.04,-6.02$, and $-2.5 \mathrm{~dB}$ ) using SA imaging relative to those obtained with conventional linear array (CLA) and plane-wave (PW) imaging techniques. Plaques were visible in all modulus elastograms, but those produced using SA and PW contained less artifacts. The modulus contrast-to-noise ratio decreased rapidly with increasing modulus contrast and attenuation coefficient, but more quickly when SA imaging was performed than for CLA or PW. The errors incurred varied from $10.9 \%$ to $24 \%(C L A), 1.8 \%$ to $12 \%(S A)$, and $\approx 4 \%(P W)$. Modulus elastograms produced with SA and $\mathrm{PW}$ imagings were not significantly different $(p>0.05)$. Despite the low transmit power, SA imaging can produce useful modulus elastograms in superficial organs, such as the carotid artery. ( $)$ The Authors. Published by SPIE under a Creative Commons Attribution 3.0 Unported License. Distribution or reproduction of this work in whole or in part requires full attribution of the original publication, including its DOI. [DOI: 10.1117/1.JMI.1.2.027001]
\end{abstract}

Keywords: elastography; shear modulus; vascular imaging; stroke; carotid artery; sparse-array.

Paper 14027PR received Mar. 18, 2014; revised manuscript received May 29, 2014; accepted for publication May 30, 2014; published online Jul. 4, 2014.

\section{Introduction}

In the United States, strokes kill over 137,000 people each year; ${ }^{1}$ these deaths occur when life-threatening plaques rupture in carotid arteries. ${ }^{2}$ Pathological studies reveal that the life-threatening plaques have distinct features: large necrotic cores, thin fibrous caps measuring $65 \mu \mathrm{m}$ or less, and are infiltrated by macrophages. ${ }^{3}$ Life-threatening plaques can evade detection because atherosclerosis may progress for several years without any symptoms. Conventional imaging techniques, such as magnetic resonance imaging (MRI), diagnostic ultrasound (US), and multislice computed tomography (CT) can evaluate the plaque burden and stenosis in symptomatic patients. ${ }^{4,5}$ However, clinicians cannot use stenosis to predict future cerebrovascular events. To predict these events, clinicians need information about the stress distribution within the fibrous cap.

The stress distribution within the fibrous cap governs the propensity of plaque to rupture. Rupture occurs when the stresses induced by the pulsating blood pressure and the hemodynamic factors exceed the tensile strength of the fibrous cap. Several studies predict that (a) stress concentrates at the junction between the fibrous cap and the normal vessel wall, ${ }^{6}$ and (b) fibrous caps rupture at locations where the tensile stress exceeds $300 \mathrm{kPa} .^{7,8}$ No imaging modality can visualize stress in vivo; however, finite element analysis can provide valuable insights into the stress distribution within the fibrous cap

*Address all correpondence to: Marvin M. Doyley, E-mail: m.doyley@ rochester .edu when the mechanical and the morphological properties of vascular tissues are known. Multislice CT and diagnostic US can visualize the morphology of carotid arteries in vivo, but they cannot measure mechanical properties. High resolution MRI can visualize plaque morphology and qualify its compositions, ${ }^{9}$ but MRI is expensive and inflexible.

Noninvasive vascular elastography (NIVE) ${ }^{10}$ is an emerging US imaging technique that visualizes the strain distribution within vascular tissues-information that is related to the external boundary conditions and the mechanical properties. ${ }^{11}$ Like its intravascular counterpart, ${ }^{12,13}$ NIVE uses the pulsating intraluminal pressure as the source of mechanical stimulation; however, unlike intravascular ultrasound elastography, it measures strain noninvasively. Schaar et al. ${ }^{13}$ demonstrated that the strain elastograms (strain images) can reveal ruptureprone regions on the fibrous cap. NIVE acquires strain elastograms from the cross-sectional plane of carotid arteries, which are difficult to interpret. This difficulty occurs because NIVE measures axial strains in the transducer's frame of reference (cartesian), which does not represent the strains in the vessel's coordinate system. To overcome this limitation, several groups, including ours, have developed strategies to estimate both the axial and the lateral components of displacements. More specifically, the proposed strategies include (a) visualizing von Mises strain $;{ }^{10}$ (b) using US beam steering techniques to measure radial and circumferential strains $;{ }^{14-18}$ (c) computing radial and circumferential strain elastograms from axial and lateral displacements measured with sparse array (SA) US imaging. ${ }^{19,20}$ 
In addition to improving NIVE strain elastograms, better estimates of lateral displacements will improve the performance of model-based elastography. ${ }^{21}$ US model-based elastography methods produce less accurate modulus elastograms than their MRI counterpart ${ }^{22}$ because only the axial component of displacement is included in the modulus recovery process. US provides imprecise estimates of the other components of displacements. $^{23}$

Our long-term goal is to develop a more quantitative approach to NIVE based on SA imaging - a technique we call quantitative sparse array vascular elastography (qSAVE). SA provides high-precision axial and lateral displacements, ${ }^{24}$ but there are concerns that the low transmit power of SA could hamper clinical use. We hypothesize that qSAVE can produce useful modulus elastograms in superficial organs, such as the carotid arteries. To corroborate this hypothesis, we assess the performance (accuracy, contrast recovery, and contrast transfer efficiency) of modulus and strain elastograms acquired with SA imaging relative to those obtained using conventional and planewave (PW) imaging.

\section{Methods}

\subsection{Soft Prior Reconstruction Method}

We used an iterative inversion scheme to compute modulus elastograms,${ }^{25-27}$ which estimates shear modulus by minimizing the difference between the measured and the computed displacements in a least squares sense. The objective function, $\Omega(\mu)$, that was minimized had the following form:

$\Omega(\mu)=\left\|\mathbf{u}_{m}-\mathbf{u}_{c}(\mu)\right\|^{2}+\alpha\left\|\mu-\mu_{0}\right\|^{2}$,

where $\mu$ is an $n \times 1$ vector of nodal shear modulus values, $n$ is the total number of nodes; $\mathbf{u}_{m}$ and $\mathbf{u}_{c}$ are the measured and the computed $2 n \times 1$ vectors of axial and lateral displacements; $\mu_{0}$ is an $n \times 1$ vector of modulus priors (i.e., a priori information about the tissue's mechanical properties); and $\alpha$ is the regularization parameter. Minimizing Eq. (1) with respect to shear modulus produced the following matrix solution at the $(k+1)$ 'th iteration:

$\mu^{k+1}=\mu^{k}+\left[\mathbf{J}^{T} \mathbf{J}+\alpha \mathbf{I}\right]^{-1} \cdot \mathbf{J}^{T}\left[\mathrm{u}_{m}-\mathrm{u}_{c}\left(\mu^{k}\right)\right]-\alpha\left(\mu^{k}-\mu_{0}\right)$,

where the matrix $\mathbf{J}$ is the $2 n \times n$ Jacobian matrix. $\mathbf{I}$ is the $n \times n$ identity matrix, and the superscript $T$ denotes the transpose operator. We computed Young's modulus (E) from Lambda modulus $(\lambda)$, shear modulus $(\mu)$, and an assumed Poisson's ratio $(\nu)$ of 0.495 . Lamè constants (i.e., $\lambda$, and $\mu$ ) are related to the Young's modulus and Poisson's ratio, as follows: ${ }^{28,29}$

$\mu=\frac{\mathbf{E}}{2(1+\nu)}, \quad \lambda=\frac{\nu \mathbf{E}}{(1+\nu)(1-2 \nu)}$.

Solving Eq. (2) may not provide unique modulus elastograms ${ }^{30}$ because the inverse elasticity problem is ill-posed; however, including geometric information in the modulus recovery process will transform the ill-posed problem to a well-posed one. ${ }^{31-35}$ We included geometric information in the image reconstruction process by minimizing the following objective function:
$\Omega(\mu)=\left\|\mathbf{u}_{m}-\mathbf{u}_{c}(\mu)\right\|^{2}+\alpha\|\mathbf{L}[\mu]\|^{2}$

where $\mathbf{L}$ is the $2 n \times n$ penalty matrix that was defined as follows: ${ }^{36}$

$L_{i, j}= \begin{cases}1 & i=j \\ 0 & i, j \notin R \\ -\frac{1}{m} & i, j \in R\end{cases}$

where $m$ is the total number of nodes contained in a given region, $R$, and $i$ and $j$ are indices of $\mathbf{L}$. The $\mathbf{L}$ matrix (i.e., Laplacian) computes the average modulus in each region explicitly as follows:

$L_{1} \cdot \mu=\mu_{1}-\frac{\mu_{2}}{N}-\frac{\mu_{3}}{N}-\cdots-\frac{\mu_{N}}{N} \approx \mu_{1}-\langle\mu\rangle$.

Minimizing Eq. (4) with respect to the shear modulus gave the following matrix solution at the $(k+1)$ 'th iteration:

$\mu^{k+1}=\mu^{k}+\left[\mathbf{J}^{T} \mathbf{J}+\alpha \mathbf{L}^{T} \mathbf{L}\right]^{-1} \cdot \mathbf{J}^{T}\left[\mathbf{u}_{m}-\mathrm{u}_{c}\left(\mu^{k}\right)\right]-\alpha \mathrm{L}^{T} \mathbf{L} \mu^{k}$.

We implemented this reconstruction method in FORTRAN 90 and compiled it on a 16-core Intel Xeon server that was running at $2.93 \mathrm{GHz}$ under the Centos 5.6 (64-bit) operating system. The reconstruction process converged to a stable solution after 10 iterations (i.e., within $5 \mathrm{~min}$ ), such that successive updates were negligible, i.e., $\left[\mathbf{J}^{T} \mathbf{J}+\alpha \mathbf{L}^{T} \mathbf{L}\right]^{-1} \cdot \mathbf{J}^{T}\left[\mathbf{u}_{m}-\mathbf{u}_{c}\left(\mu^{k}\right)\right]-$ $\alpha \mathrm{L}^{T} \mathrm{~L} \mu^{k} \approx 0$.

\subsection{Ultrasound Imaging}

We performed conventional linear array (CLA) imaging with 64 active transmission elements and 32 reception elements- the default configuration for the US scanner used in our experimental study. We applied delays during the transmission to focus the US beam and a Hanning apodization function to the received echoes. Beam-forming was performed with the delay-sum technique. The lateral sampling frequency of all beam-formed radiofrequency (RF) echo frames was increased from 0.30 to 52 lines/mm using the method described in Konofagou and Ophir. $^{37}$

We performed SA imaging by transmitting on 15 elements sequentially, which spanned the full length of the array and received on all 128 elements. Like CLA imaging, all RF echo frames were beam-formed using the delay-sum technique. Dynamic focusing was performed both on transmission and reception. The received signals were apodized with a boxcar function. $^{24}$

We performed PW imaging by transmitting and receiving on all 128 elements. Beam-forming was also performed with the delay-sum technique.

\subsection{Beam-Forming}

RF echo frames were reconstructed by applying the delay-sum technique to CLA, SA, and PW data. The backscatter signal at point $\left(x_{0}, z_{0}\right)$ in the image was reconstructed as follows:

$s\left(x_{0}, z_{0}\right)=\sum_{i=1}^{N_{t x}} \sum_{j=1}^{N_{r x}} w_{i j} R F_{i j}\left[t-\tau\left(x_{0}, z_{0}\right)\right]$, 
where $N_{t x}$ and $N_{r x}$ represent the number of transmit and receive elements in the linear array; $R F_{i j}(t)$ represents the $\mathrm{RF}$ echo when the $i$ 'th element transmits and the $j$ 'th element receives; $t$ represents the time of flight of the echo; $\tau\left(x_{0}, z_{0}\right)$ represents the round-trip time from point $\left(x_{0}, z_{0}\right) ; w_{i j}$ represents the apodization weight. During SA imaging, only a few elements in the array were active during transmission, but all the elements were active during reception. We used a transmit-receive (T/ R) matrix to compute the apodization weights $w_{i j}$ that produced beam patterns similar to those generated with a fully populated array. ${ }^{24,38,39}$ The apodization weights employed in PW imaging were identical to those employed in a fully populated array because all 128 elements were active during transmission and reception. The time, $\tau_{t x}$, required to reach a given point $\left(x_{0}, z_{0}\right)$ was computed as follows:

$\tau_{t x}=\left[\sqrt{\left(x_{i}-x_{0}\right)^{2}+z_{0}^{2}}\right] / c$,

where $x_{i}$ represents the location of the transmitting element and $c$ represents the speed of sound. During PW imaging, the time required for the US beam to travel to a given point was computed as follows:

$\tau_{t x}=\frac{z_{0}}{c}$

For all imaging techniques, the return-trip time $\left(\tau_{r x}\right)$ was computed as follows:

$\tau_{r x}=\left[\sqrt{\left(x_{j}-x_{0}\right)^{2}+z_{0}^{2}}\right] / c$,

where $x_{j}$ represents the location of the receiving element. The total round-trip time to and from the point $\left(x_{0}, z_{0}\right)$ was computed as follows:

$\tau\left(x_{0}, z_{0}\right)=\tau_{t x}+\tau_{r x}$.

SA and PW RF echo frames were reconstructed on a $10 \times 10 \mathrm{~mm}^{2}$ grid that had a lateral sampling frequency of 52 lines/mm and an axial sampling frequency of $40 \mathrm{MHz}$. The axial sampling frequency of beam-formed conventional RF echo frames was similar to those of SA and PW echo frames; however, the lateral sampling frequency was lower than 0.3 lines $/ \mathrm{mm}$.

\subsection{Strain and Displacement Estimation}

We created axial and lateral displacement elastograms by applying a two-dimensional (2-D) cross-correlator ${ }^{19,24}$ to the pre- and postdeformed RF echo frames. All cross-correlation analyses were performed with $2 \times 2 \mathrm{~mm}^{2}$ kernels that overlapped by $80 \%$ in both the axial and lateral direction. We used a $5 \times 5$ median filter to remove spurious displacement estimates.

\subsection{Data Analysis}

We used the elastographic contrast-to-noise ratio $\left(\mathrm{CNR}_{e}\right)$ and the normalized root-mean-square performance metric to assess the performance of both strain and modulus elastograms. The elastographic CNR metric was defined as follows: ${ }^{40}$
$\mathrm{CNR}_{e}[\mathrm{~dB}]=20 \log \frac{2\left(\theta_{w}-\theta_{p}\right)^{2}}{\left(\sigma_{w}^{2}+\sigma_{p}^{2}\right)}$

where $\theta_{w}$ and $\theta_{p}$ represent the mean strain or modulus in the vessel wall and plaque; and $\sigma_{w}$ and $\sigma_{p}$ represent the standard deviation in strain or modulus values in the corresponding regions.

The normalized root-mean-squared error (NRMSE) was computed as follows:

$\mathrm{NRMSE}=\frac{\sqrt{\frac{\sum_{i=1}^{N}\left[x_{m}(i)-x_{c}(i)\right]^{2}}{N}}}{\max \left[x_{c}\right]-\min \left[x_{c}\right]}$,

where $x_{c}$ and $x_{m}$ represent the actual and measured parameters (modulus or strain), respectively, and $N$ is the number of pixels in the region of interest.

\section{Simulation Study}

The goal of this simulation study was to corroborate the hypothesis that qSAVE can recover useful modulus elastograms from attenuating materials. We compared the quality of modulus elastograms reconstructed from axial and lateral displacements measured from vessel phantoms with varying modulus contrasts $(-12.04,-6.02$, and $-2.50 \mathrm{~dB})$ and attenuation coefficients $(0.6,1$, and $3.5 \mathrm{~dB} / \mathrm{cm})$. Axial and lateral displacements were computed by applying our 2-D echo tracking technique to RF echo frames acquired with (a) CLA imaging with lateral interpolation, (b) SA imaging, and (c) PW imaging. For the reminder of this manuscript, we will refer to the first approach as CLA imaging. We used a two-step process to synthesize RF echo frames. In the proceeding subsections, we describe (1) the two key stages of the simulation process: mechanical (solving the forward elasticity problem) and acoustic modeling; and (2) the modulus reconstruction protocol.

\subsection{Mechanical Model}

We used a commercially available finite element package (ABAQUS; Dassault Systems; Velizy-Villacaublay, France) to create three finite element models of diseased arteries. The simulated arteries were incompressible $(\nu \approx 0.495)$ with inner and outer radii of 1.5 and $6 \mathrm{~mm}$, respectively. We assigned a Young's modulus of $50 \mathrm{kPa}$ to the vessel wall, but varied the modulus of the simulated plaques to generate vessel phantoms

Table 1 Composition of materials used to fabricate vessel phantoms.

\begin{tabular}{lccccc}
\hline & & & & \\
& $\begin{array}{c}\text { PVA } \\
\%\end{array}$ & $\begin{array}{c}\mathrm{Al}_{2} \mathrm{O}_{3} \\
\%\end{array}$ & $\begin{array}{c}\mathrm{SiC} \\
\%\end{array}$ & $\begin{array}{c}\text { Water } \\
18 \mathrm{M} \Omega \\
(\%)\end{array}$ & $\begin{array}{c}\text { Thermal } \\
\text { cycles }\end{array}$ \\
\hline Phantom \#1: plaque & 8 & 0 & 3 & 89 & 2 \\
$\begin{array}{l}\text { Phantom \#1: vessel } \\
\text { wall }\end{array}$ & 8 & 0 & 1 & 91 & 5 \\
$\begin{array}{l}\text { Phantom \#2: plaque } \\
\begin{array}{l}\text { Phantom \#2: vessel } \\
\text { wall }\end{array}\end{array}$ & 8 & 5 & 2 & 85 & 2 \\
\hline
\end{tabular}


(a)

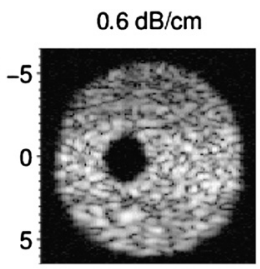

(b)

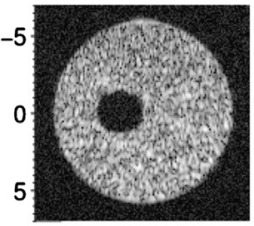

(c)

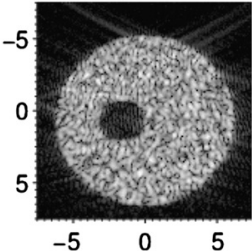

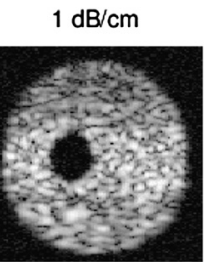
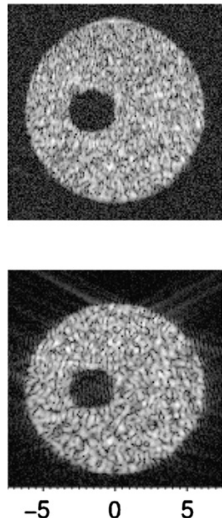

$3.5 \mathrm{~dB} / \mathrm{cm}$
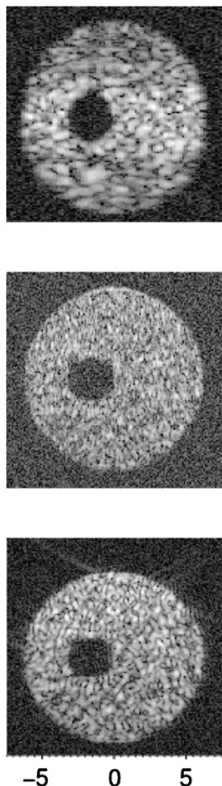

Fig. 1 Sonograms obtained using (a) conventional linear array (CLA), (b) sparse-array (SA), and (c) plane-wave (PW) imaging from simulated vessels with attenuation coefficients of $0.6,1$, and $3.5 \mathrm{~dB} / \mathrm{cm}$ going from left to right.

with modulus contrasts of $-12.04,-6.02$, and $-2.50 \mathrm{~dB}(12.5$, 25.0 , and $37.5 \mathrm{kPa})$. These mechanical parameters were representative of those reported in Ref. 16. A uniformly distributed pressure $(666.7 \mathrm{~Pa})$ was applied to the inner lumen, which generated a maximum strain of $1 \%$. To minimize rigid body motion, we constrained the motion of two nodes on the inner and outer boundaries. More specifically, one node on the outer boundary was not allowed to move in the circumferential direction, while the node directly across from it on the inner boundary was not permitted to move radially.

\subsection{Acoustic Model}

We used the Field $\mathrm{II}^{41}$ simulation environment to compute the acoustic response of the L14-5/38 linear transducer array (Prosonic Corporation, Korea) used in the experimental studies when operating at $5 \mathrm{MHz}$. We simulated the acoustic response of the predeformed vessel by randomly distributing point scatterers (16 scatterers per wavelength) within the simulated vessels. ${ }^{42}$ The speed of sound in the vessel models was set to $1540 \mathrm{~m} \mathrm{~s}^{-1}$. Solving the forward elasticity problem produced displacements that were used to redistribute the point scatterers of the predeformed vessels (i.e., compute the acoustic response of the postdeformed vessel). We simulated RF echo frames for vessels with attenuation coefficients of $0.6,1.5$, and $3.5 \mathrm{~cm}^{-1}$. We used an additive Gaussian white noise model to simulate RF echo frames with signal-to-noise ratios (SNRs) of $26 \mathrm{~dB}$ (CLA), $20 \mathrm{~dB}(\mathrm{SA})$, and $29 \mathrm{~dB}(\mathrm{PW})$. These SNR values represent those that were measured experimentally when our US system was
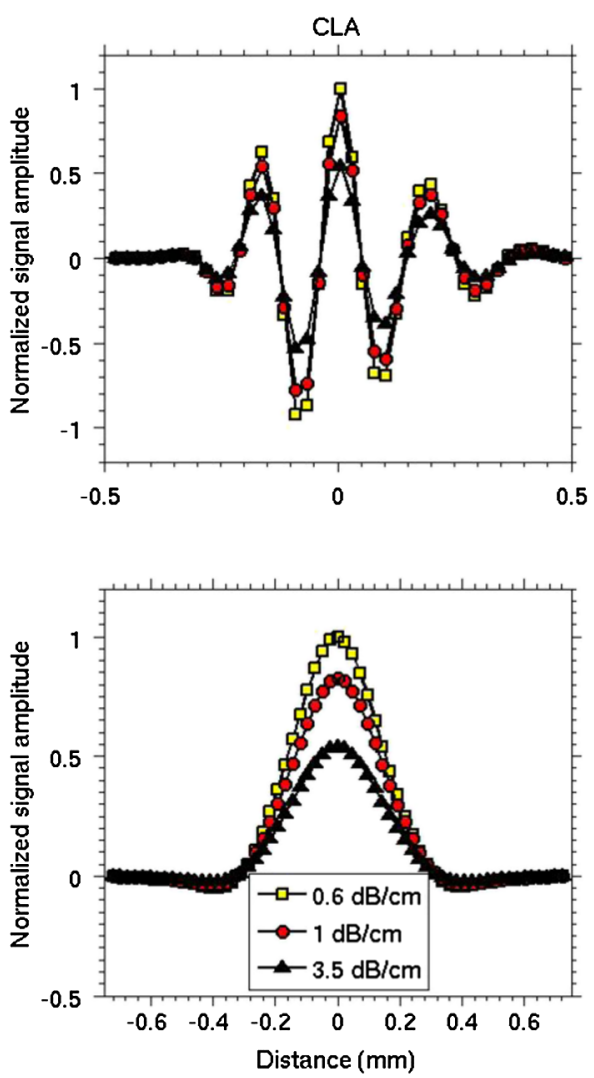

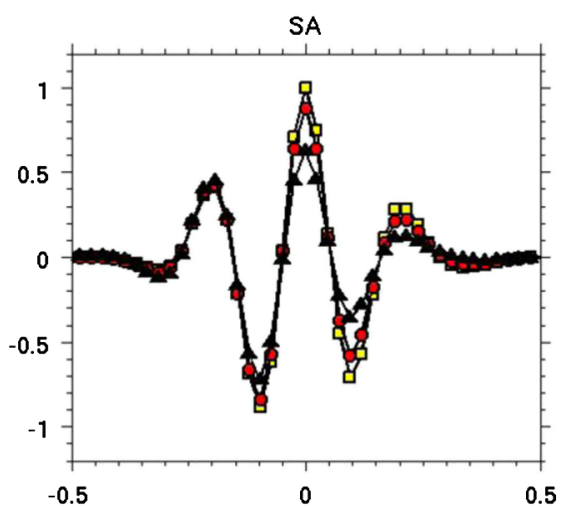

(a)

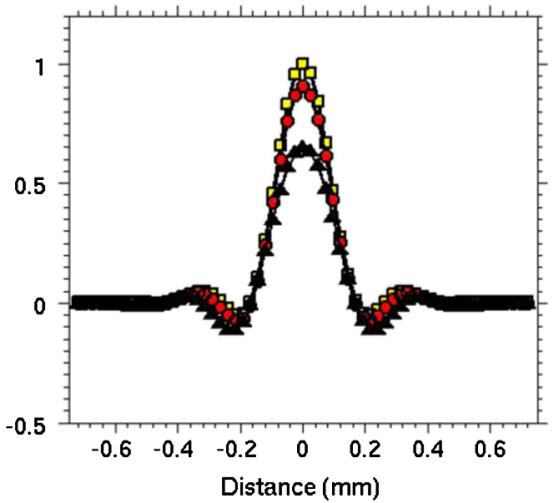

(b)
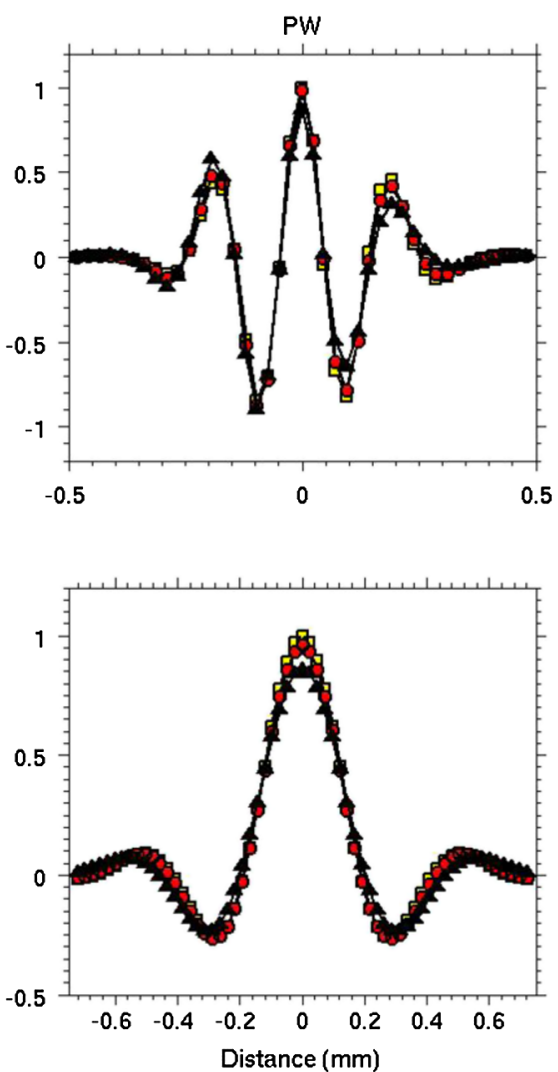

Fig. 2 (a) Axial and (b) lateral profiles taken through the center of point spread functions for CLA, SA, and PW imaging systems. 
configured to acquired RF echo frames with CLA, SA, and PW imagings.

To assess the variability of our reconstruction approach, we performed 25 statistically independent reconstructions at each contrast and attenuation coefficient.

\subsection{Modulus Reconstructions}

All modulus elastograms were reconstructed from a homogeneous shear modulus distribution of $16.67 \mathrm{kPa}$. Structural information was obtained by manually segmenting either the modulus distribution used in the finite element model or sonograms. All reconstructions were performed using a homogeneous finite element mesh consisting of 7,475 triangular elements and 3,910 nodes. Reconstructions were performed with three different values of the regularization parameter. More specifically, the regularization parameter was set to $7.2 \times 10^{-9}, 5 \times 10^{-10}$, and $1.2 \times 10^{-9}$ when reconstruction was performed using displacements measured with CLA,
SA, and PW imagings, respectively. We used the L-curve method $^{35,43}$ to select the optimum regularization parameter objectively.

\section{Phantom Study}

\subsection{Phantom Fabrication}

We fabricated two vessel phantoms (12-mm outer diameter by 100-mm long) from a suspension of polyvinyl alcohol (PVA, Elvanol 71-30, Dupont, Wilmington, Delaware), ultra-fine aluminum oxide $(0.3 \mu \mathrm{m}$, Logitech Ltd., Glasgow, Scotland, UK), and silicon carbide (320 Grit, Fisher Scientific, Fair Lawn, New Jersey). We used aluminum oxide $\left(\mathrm{Al}_{2} \mathrm{O}_{3}\right)$ and silicon carbide (SiC) to control the attenuation coefficient ${ }^{44}$ and the echogenicity of the vessels, respectively. Table 1 shows the composition of each phantom. We used a highly controlled and repeatable process to fabricate all phantoms. ${ }^{35,45,46}$ More specifically, we placed two off-center rods (3.12-mm diameters) in a cylindrical mold

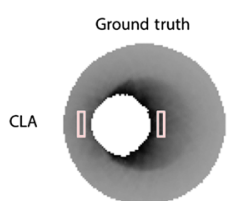

SA

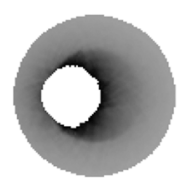

PW

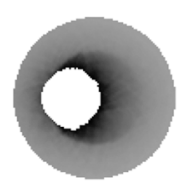

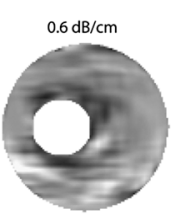
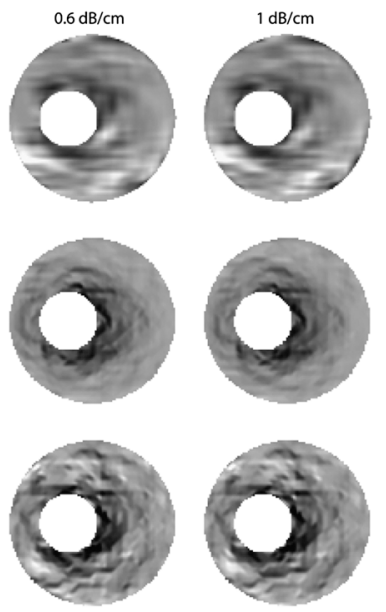

(a)
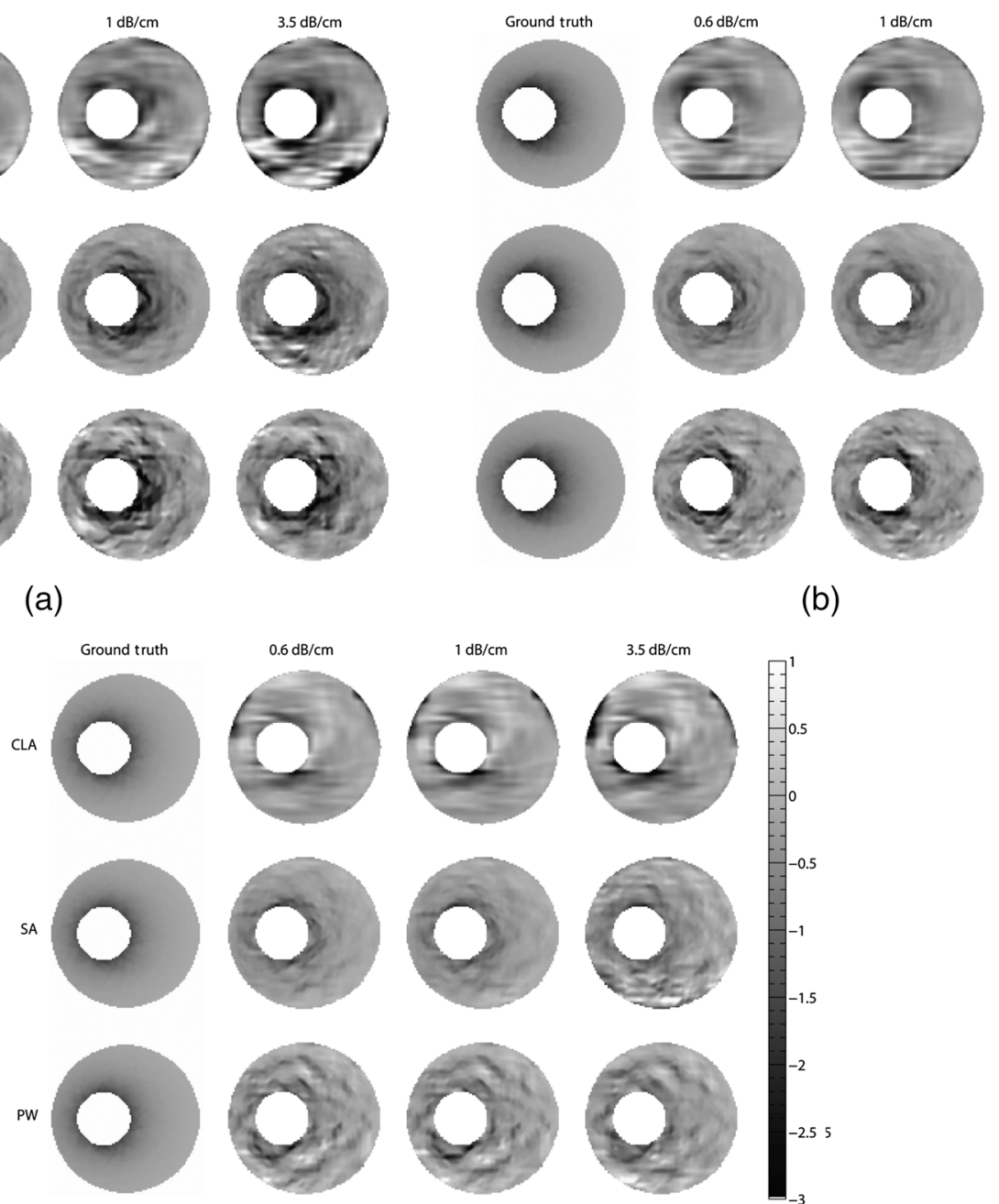

(b)

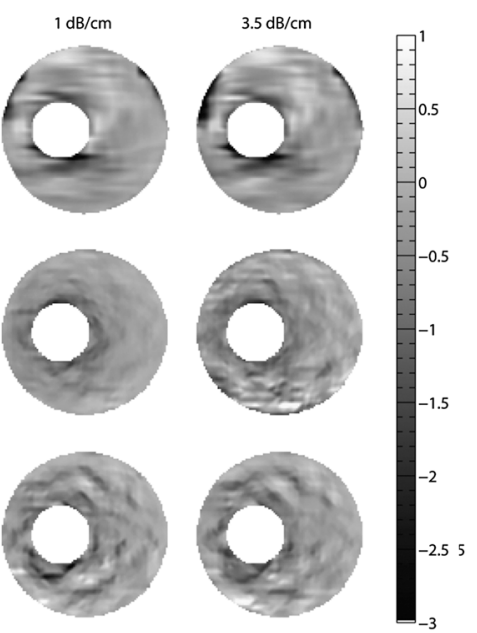

(c)

Fig. 3 Montage of radial strain elastograms, displayed in percentage, obtained from simulated vessels with modulus contrasts of (a) $-12.04 \mathrm{~dB}$, (b) $-6.02 \mathrm{~dB}$, and (c) $-2.5 \mathrm{~dB}$. Ideal strain elastograms are shown in the first column of each montage, and the elastograms obtained from vessel phantoms with attenuation coefficients of $0.6,1$, and $3.5 \mathrm{~dB} / \mathrm{cm}$ are shown in the remaining columns. 
(12-mm diameter by $120-\mathrm{mm}$ long). One rod was circular whereas the other was semicircular. Molten PVA was poured in the vacant cavity between the rods and the molds. We constructed the vessel wall by subjecting the sealed mold to three freeze-thaw cycles. One thermal cycle was completed in $24 \mathrm{~h}$ where the temperature was varied from $+20^{\circ} \mathrm{C}$ to $-20^{\circ} \mathrm{C}$. We removed the semicircular rod after thermal cycling and filled the vacant cavity with the PVA and subjected the phantom to two additional freeze-thaw cycles. After thermal cycling, the phantom was removed from the mold and stored at room temperature in water.

\subsection{Elastographic Data Acquisition}

The equipment used for elastographic imaging consisted of a Sonix RP US system (Ultrasonix, Peabody, Massachusetts) that was equipped with a 128 element linear transducer array
(L14-5/38 probe), a multichannel data acquisition system (Sonix DAQ®, Ultrasonix, Peabody, Massachusetts), a simple water column system, and a pressure wire (Millar Instruments Mikro-Cath, Houston, Texas).

We configured the Sonix RP US scanner to acquire (a) conventional US echo images (an aperture consisting of 64 transmission elements and 32 reception elements), (b) SA echo data (15 transmission elements sequentially and 128 reception elements), and (c) PW images (128 active transmission and reception elements). All echo imaging was performed at $5 \mathrm{MHz}$, and the received signal was sampled to 12 bits at $40 \mathrm{MHz}$. We used a simple water column system to pressurize the vessels to $667 \mathrm{~Pa}$. All three data sets were collected with interleaved US scanning, which we implemented using a software development kit (TEXO SDK, v5.7.1, Ultrasonix, Peabody, Massachusetts). The delay-sum technique was used to beam-form SA and PW data.

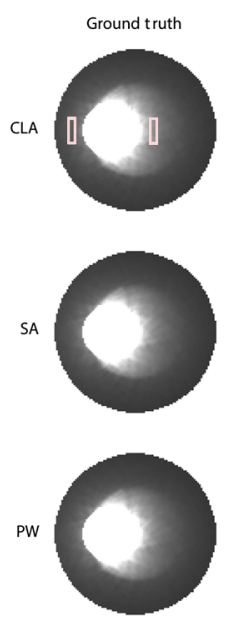

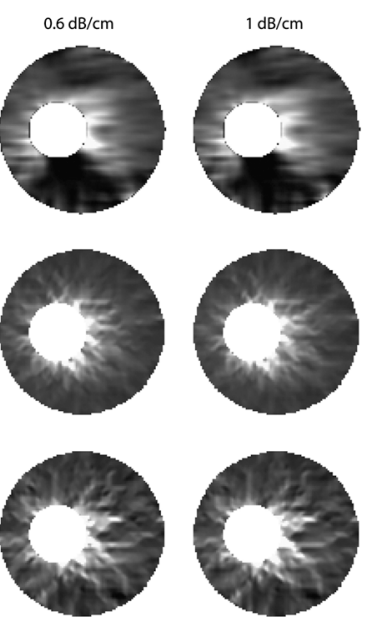

(a)
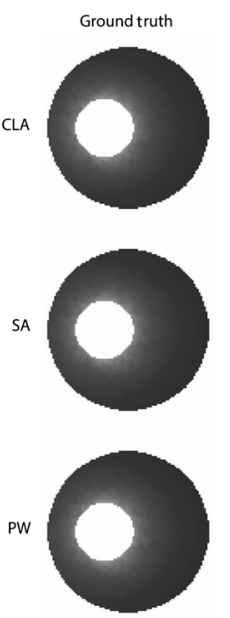
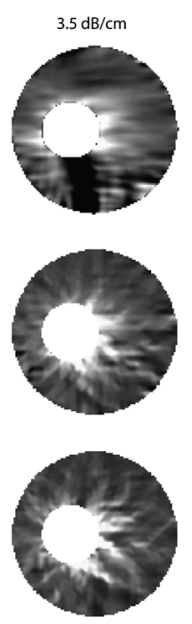

$0.6 \mathrm{~dB} / \mathrm{cm}$
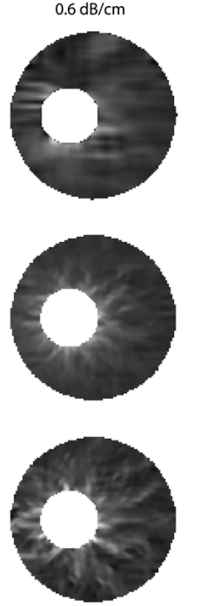
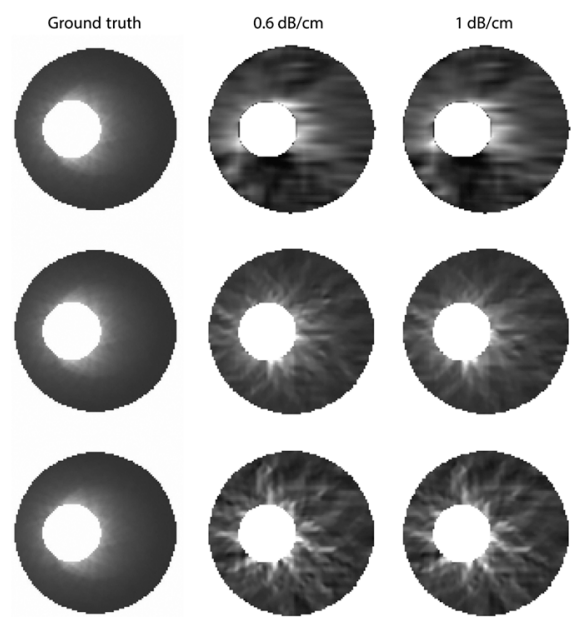

(b)
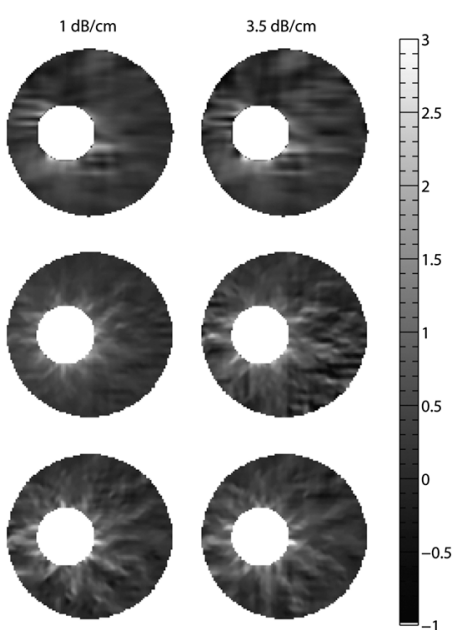

(c)

Fig. 4 Montage of circumferential strain elastograms, displayed in percentage, obtained from simulated vessels with modulus contrasts of (a) $-12.04 \mathrm{~dB}$, (b) $-6.02 \mathrm{~dB}$, and (c) $-2.5 \mathrm{~dB}$. Ideal circumferential strain elastograms are shown in the first column of each montage, and the elastograms obtained from vessel phantoms with attenuation coefficients of $0.6,1$, and $3.5 \mathrm{~dB} / \mathrm{cm}$ are shown in the remaining columns. The two rectangular boxes in (a) denotes the regions of interests corresponding to the plaque and vessel wall that was used to compute $\mathrm{CNR}_{e}$ values reported in Fig. 7. 


\subsection{Modulus Reconstructions}

Finite element representations of the vessel phantoms were computed by applying the Delaunay triangulation method (COMSOL Inc., Burlington, Massachusetts) to manually segmented sonograms. A typical mesh consisted of 7,500 nodes and 15,000 triangular elements. The pressure on the inner lumen was measured with a pressure wire during the elastographic imaging. We interpolated the measured displacements to the nodal coordinates of all the finite element meshes using a cubic interpolation function. The boundary conditions used in the phantom were similar to those used in the simulated study. All reconstructions were performed using a homogeneous shear modulus of $16.67 \mathrm{kPa}$. The regularization parameters were identical to those used in the simulation study.

\subsection{Measuring the Attenuation Coefficient}

We measured the attenuation coefficient of representative cylindrical-shaped samples of each tissue component at room temperature. All attenuation measurements were performed at $5 \mathrm{MHz}$ as follows: ${ }^{47}$

$\alpha=\frac{1}{d} \cdot 10 \log 10\left(\frac{P_{p}}{P_{w}}\right)$, where $P_{w}$ and $P_{p}$ represent the power received from water and the test sample, respectively. $d$ was the thickness of the test sample.

\section{Results}

\subsection{Simulation Study}

Figure 1 shows examples of sonograms acquired from simulated vessel phantoms (attenuation coefficients of 0.6, 1, and $3.5 \mathrm{~dB} / \mathrm{cm}$ ) with CLA, SA, and PW. Attenuation degraded the quality of all sonograms, but more quickly when SA imaging was performed. This occurred because less acoustic power was transmitted during SA imaging. PW sonograms contained visible side-lobes because no focusing was applied during transmission.

Figure 2 shows axial and lateral profiles obtained from CLA, SA, and PW point-spread functions (PSFs) when imaging was performed in mediums with attenuation coefficients of $0.6,1$, and $3.5 \mathrm{~dB} / \mathrm{cm}$. The axial profiles were similar because identical transmission frequencies were employed in all three imaging approaches. The axial profiles were oscillatory and their amplitudes decreased with increasing attenuation. However, the lateral profiles were different. SA and CLA imagings had the narrowest and broadest lateral profiles (beamwidth), respectively. The lateral profiles of SA and PW PSFs contained side-
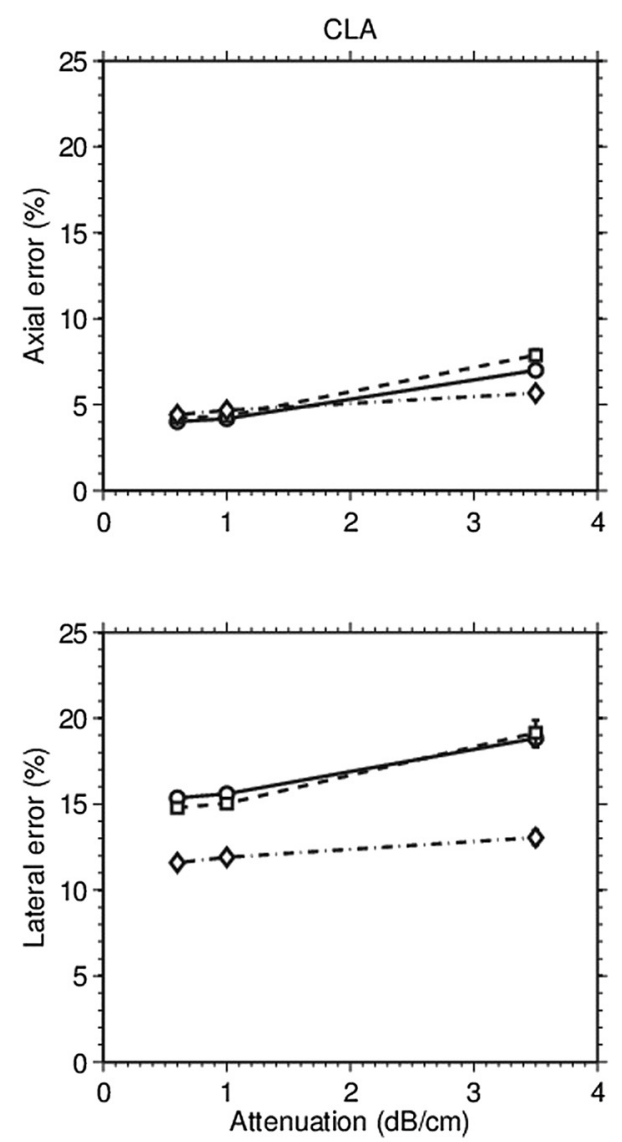

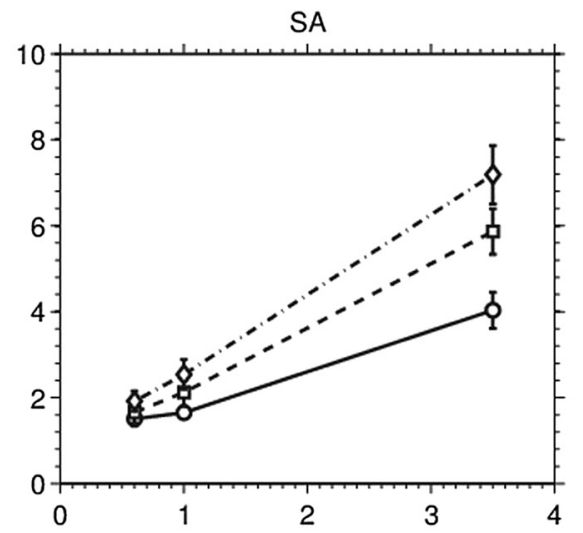

(a)

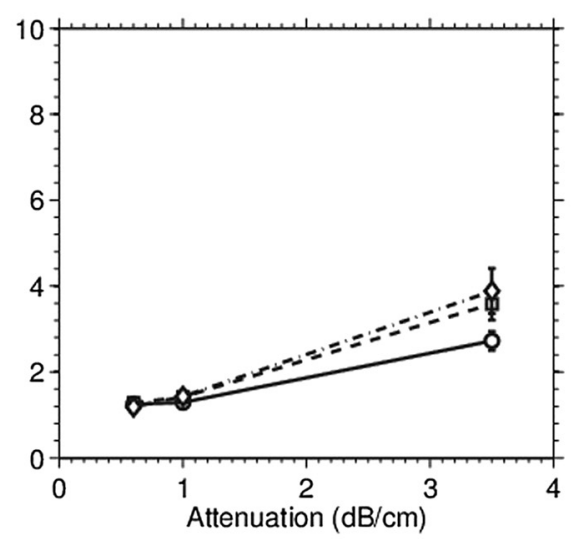

(b)
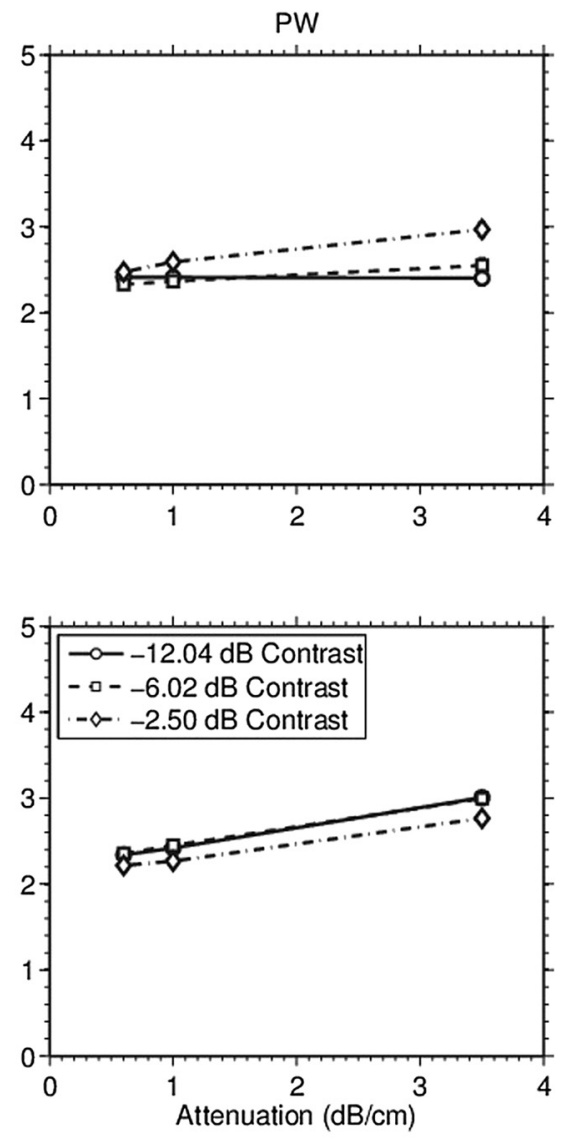

Fig. 5 The normalized root-square-mean-error incurred when estimating (a) axial and (b) lateral displacements with conventional linear array (CLA) (first column), SA (second column), and PW (third column) imagings. 
lobes; however, those obtained from the CLA did not. The box car apodization function and the larger transmission apertures employed during SA and PW imagings were responsible for the observed side-lobes.

Figures 3 and 4 show radial and circumferential strain elastograms acquired from simulated phantoms with modulus contrast of $-6.02 \mathrm{~dB},-12.04 \mathrm{~dB}$, and $-2.5 \mathrm{~dB}$ with CLA, SA, and PW imagings. Increasing the attenuation coefficient from 0.6 to $3.5 \mathrm{~dB} / \mathrm{cm}$ degraded the quality of all strain elastograms.

Figure 5 shows the errors incurred when estimating axial and lateral displacements with CLA, SA, and PW imaging plotted as a function of attenuation coefficients and modulus contrast. The accuracy of axial displacement elastograms measured with CLA and SA imagings depended on both the attenuation coefficient and the modulus contrast imaging. More specifically, the accuracy of SA axial displacement elastograms varied from $1.8 \%$ to
$7 \%$ for the range of modulus contrast and attenuation coefficients explored in this study; whereas, the accuracy of CLA axial displacement elastograms varied from $4.9 \%$ to $8 \%$ for a similar range of modulus contrast and attenuation. The accuracy of PW axial displacement elastograms was marginally affected $(\approx 2.5 \%)$ by attenuation and modulus contrast. Errors incurred when measuring lateral displacement displayed a similar trend. More specifically, the accuracy of lateral displacement elastograms varied from $12 \%$ to $20 \%$ (CLA), $1.8 \%$ to $3.9 \%$ (SA), and $2.2 \%$ to $3 \%(\mathrm{PW})$ for the range of attenuation coefficients and modulus contrasts explored in this study.

Figure 6 shows the corresponding modulus elastograms reconstructed from the axial and lateral displacement elastograms measured with CLA, SA, and PW imagings (i.e., Fig. 4). All modulus elastograms revealed the plaque, but SA and PW modulus elastograms contained less artifacts.

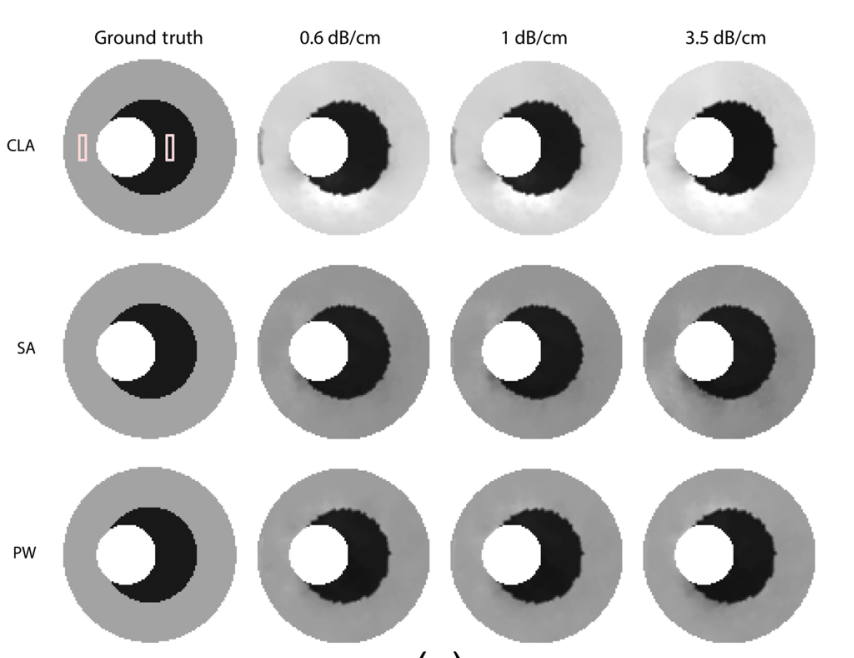

(a)
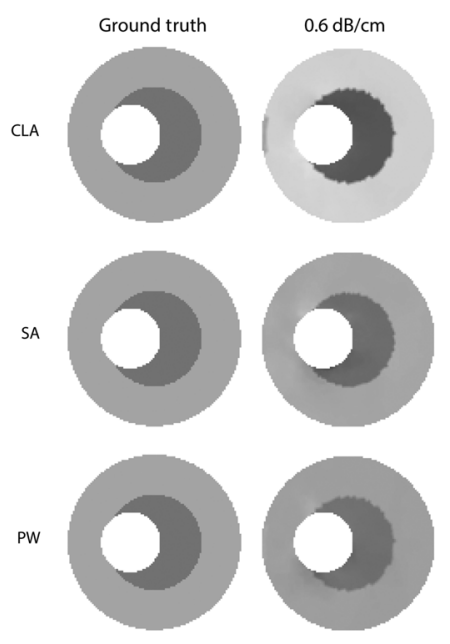

(c)
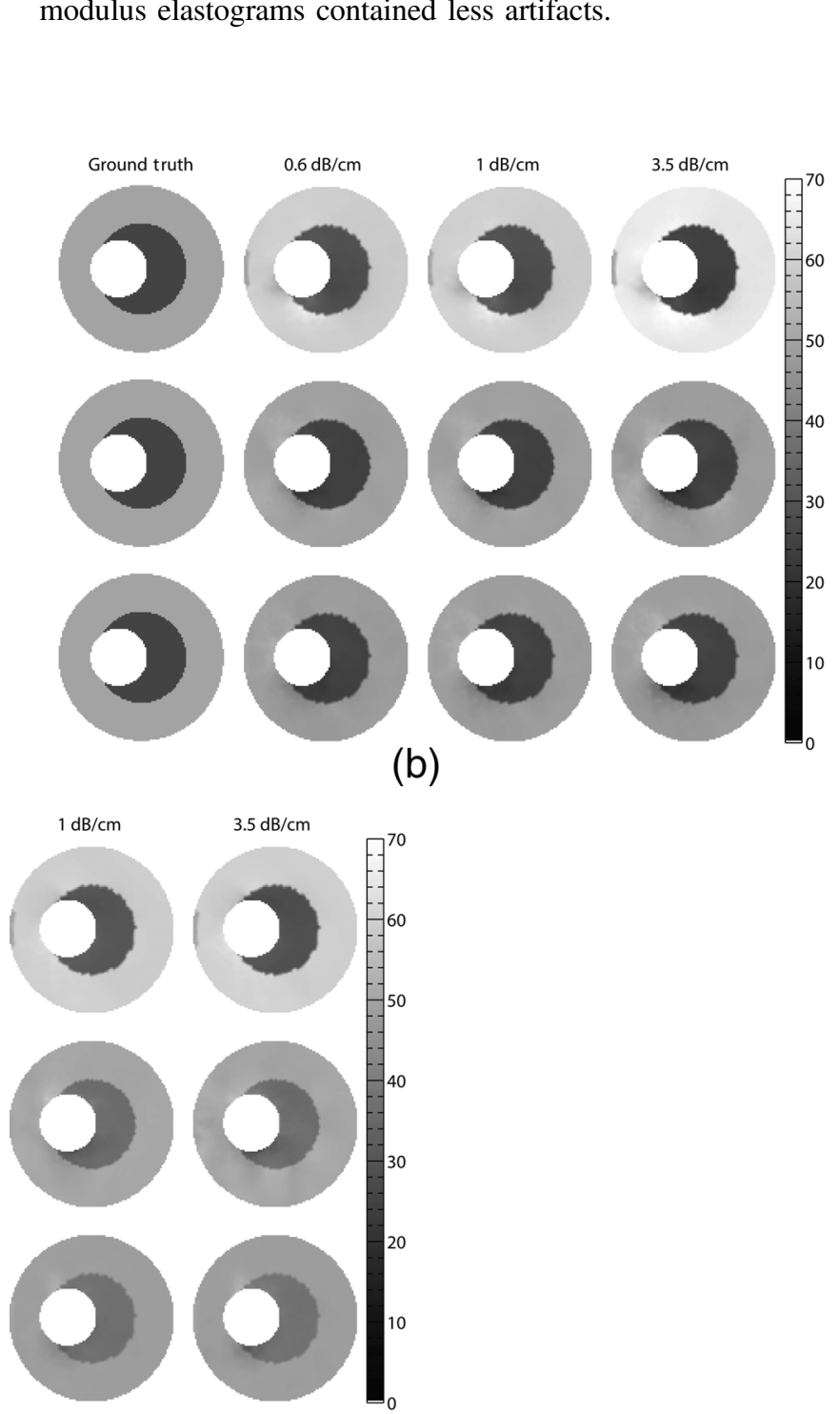

(b)

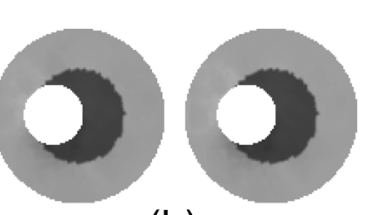

\section{.

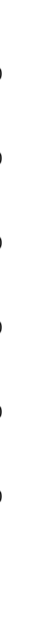

Fig. 6 Montage of modulus elastograms, displayed in units of kilo Pascal $(\mathrm{kPa})$, corresponding to the strain elastograms shown in Figs. 3 and 4 . The first column of each montage shows the actual modulus elastograms and the remaining columns show the elastograms obtained from the simulated vessels with modulus contrasts of (a) $-12.04 \mathrm{~dB},(\mathrm{~b})-6.02 \mathrm{~dB}$, and (c) $-2.5 \mathrm{~dB}$. Showing (a) conventional, (b) $\mathrm{SA}$, and (c) PW modulus elastograms. The two rectangular boxes in (a) denotes the regions of interests corresponding to the simulated plaque and vessel wall that was used to compute $\mathrm{CNR}_{e}$ values reported in Fig. 7. 
Figure 7 shows bar plots of the recovered contrast and the accuracy of modulus elastograms. The image reconstruction process recovered the modulus contrast more accurately when it was applied to displacements measured with SA and PW than those measured with CLA. For CLA and SA imaging, the variance of the recovered modulus contrast increased rapidly with attenuation; however, variance increased slightly with increasing attenuation and modulus contrast during PW imaging. Modulus elastograms computed with SA and PW were more accurate than those computed with CLA. More specifically, the accuracy of modulus elastograms varied from $17 \%$ to $26 \%$ (CLA), $2 \%$ to $10 \%$ (SA), and $4 \%$ to $5 \%$ (PW) over the range of modulus contrast and attenuation explored in this work.

Figure 8 shows the $\mathrm{CNR}_{e}$ computed from strain and modulus elastograms (Figs. 4-6) plotted as a function of attenuation coefficients and modulus contrasts. Both types of elastograms (i.e., strain and modulus) displayed a similar trend. $\mathrm{CNR}_{e}$ decreased with increasing attenuation and increased with raising the modulus contrast; however, the $\mathrm{CNR}_{e}$ of strain elastograms were lower than those computed from modulus elastograms. This was due to differences in spatial resolution of modulus and strain imaging. ${ }^{48}$ If spatial resolution of the images are similar, then both images should have the same $\mathrm{CNR}_{e}$; however, equalizing the spatial resolution of modulus and strain elastograms is not trivial given the complexity of the reconstruction process.

\subsection{Phantom Study}

Figures 9-i and 9-iv show representative examples of sonograms obtained from vessel phantoms with the three imaging methods (CLA, SA, and PW). Side-lobes were discernible in the PW sonograms, which was expected because no focusing was performed during transmission.

Figures 9-ii and 9-v show example correlation images (i.e., the peak correlation coefficient $(\rho)$ obtained at each pixel during echo tracking). When elastographic imaging was performed on the phantom with the lower attention coefficient $(0.6 \mathrm{~dB} / \mathrm{cm})$, most of the pixels in CLA and SA correlation images exceeded the threshold (i.e., $\rho \geq 0.90$ ) required to estimate displacement precisely. The higher side-lobe level in the PW images is responsible for the lower cross-correlation coefficient (decreased performance). This trend was reversed when elastography was performed on the higher attenuating phantom, which was not surprising because PW sonograms had considerably higher sonographic SNR than either CLA or SA.

Figures 9-iii and 9-vi show examples of modulus elastograms recovered from the attenuating phantoms with CLA, SA, and PW imagings. The plaque was visible in all modulus elastograms as a localized region of low elasticity. Table 2 summarizes the mean modulus recovered from the plaque and vessel wall for each phantom, which demonstrates that the SA and the PW modulus elastograms were more accurate than those
CLA
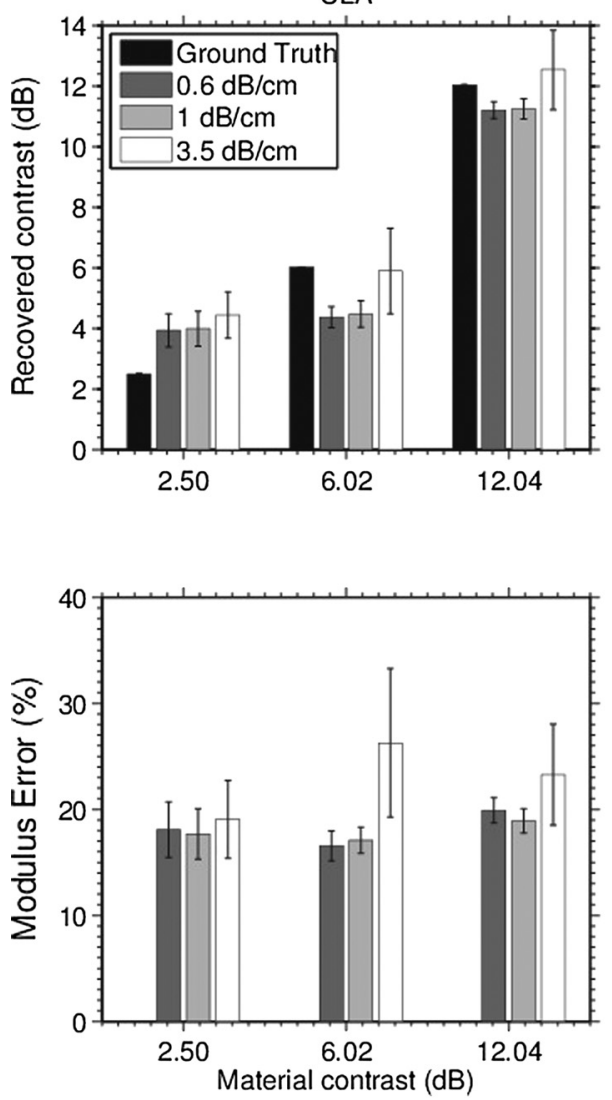

SA

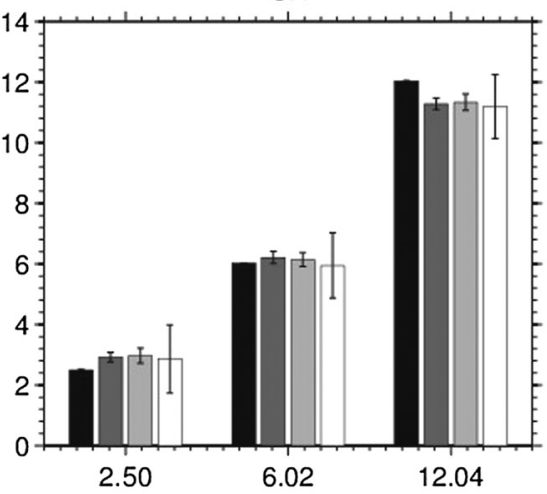

(a)

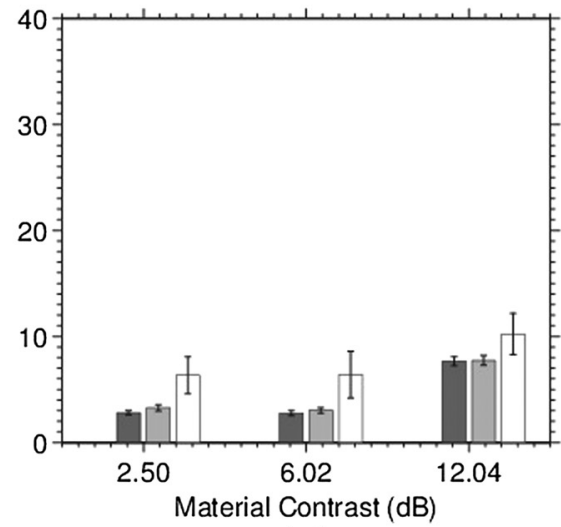

(b)
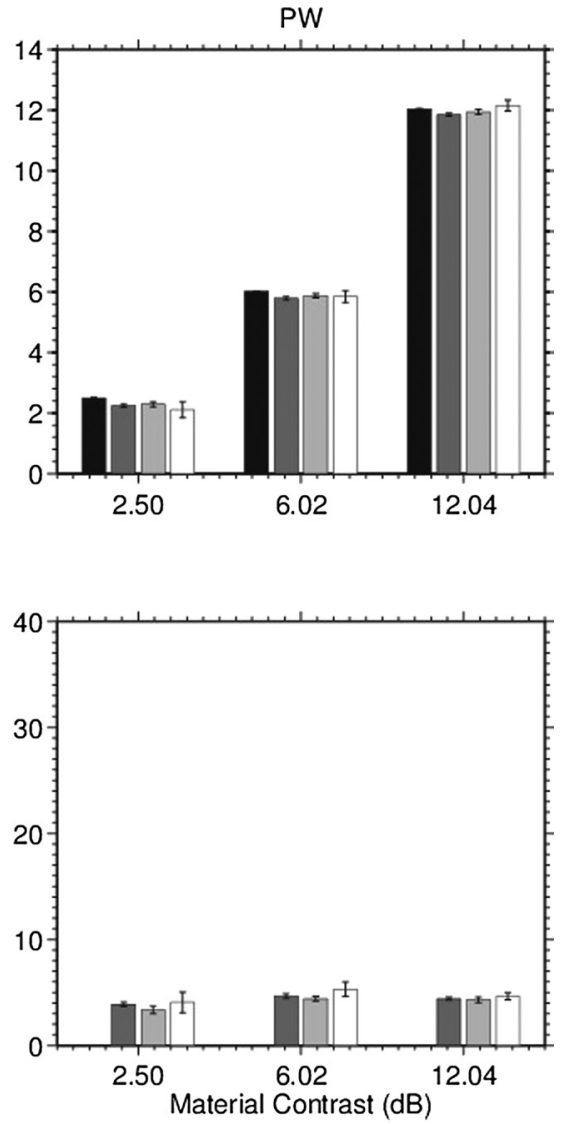

Fig. 7 (a) Recovered modulus contrast and (b) modulus error incurred when elastography imaging was performed with CLA (left-column), SA (middle-column), and PW (right-column) imaging. 
produced with CLA. Figure 10 shows bar plots of accuracy and $\mathrm{CNR}_{e}$ of the recovered modulus elastograms. $\mathrm{CNR}_{e}$ of $\mathrm{SA}$ and PW modulus elastograms were higher than those of CLA modulus elastograms, which was consistent with the results of the simulation study. In general, the contrast recovered from SA and PW modulus elastograms was better than those obtained from CLA elastograms.

\section{Discussion}

In this study, we compared the performance of SA elastograms (displacements, strain, and modulus) to those measured with CLA and PW imagings. The primary findings of this study were as follows: (a) SA and CLA produced the narrowest and broadest US beams, respectively (Fig. 2); (b) increasing modulus contrast and attenuation coefficients reduced the accuracy of axial and lateral displacement elastograms measured with SA, but marginally affected those produced with CLA and PW imaging (Fig. 5); (c) all imaging methods produced stable modulus elastograms (Figs. 6 and 9) whose variance increased linearly with raising attenuation (Fig. 7); (d) the accuracy of modulus elastograms varied from $18 \%$ to $24 \%$ (CLA), $2 \%$ to $12 \%$ (SA), and $3 \%$ to $4 \%$ (PW).

Lateral sampling frequency of the US echo frames influenced the precision of lateral displacements (Fig. 3), which is congruent with results reported in Konofagou and Ophir. ${ }^{49}$ Sampling frequencies higher than 52 lines/mm had little effect on performance (not shown), because there is a limit as to how much lateral sampling can improve precision. ${ }^{24,49}$ In addition to lateral sampling frequency, the transmit and receive apertures also impact the precision of lateral displacements. More specifically, larger apertures produce tighter beams than smaller ones that improve precision, but at the expense of side-lobes ${ }^{50}$ (Fig. 2). The CLA imaging produced the widest beam (beam-width of $0.31 \mathrm{~mm}$ ) because a smaller aperture (11-mm transmission
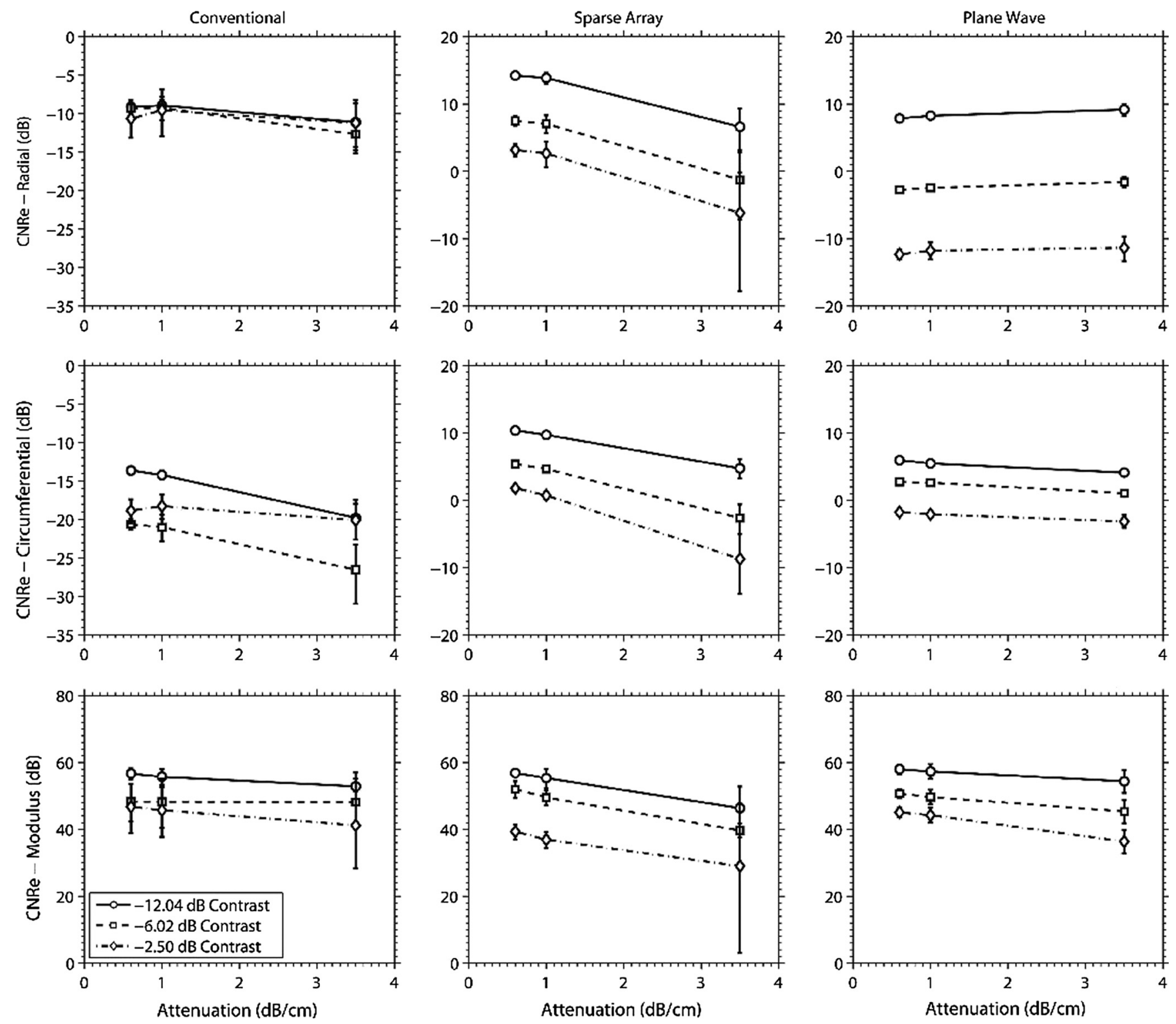

Fig. 8 The contrast-to-noise ratio of strain (top and middle rows) and modulus (bottom rows) elastograms obtained from simulated vessels with modulus contrast of $-2.5 \mathrm{~dB},-6.02 \mathrm{~dB}$, and $-12.04 \mathrm{~dB}$, and attenuation coefficients of 0.6 to $3.5 \mathrm{~dB} / \mathrm{cm}$. 

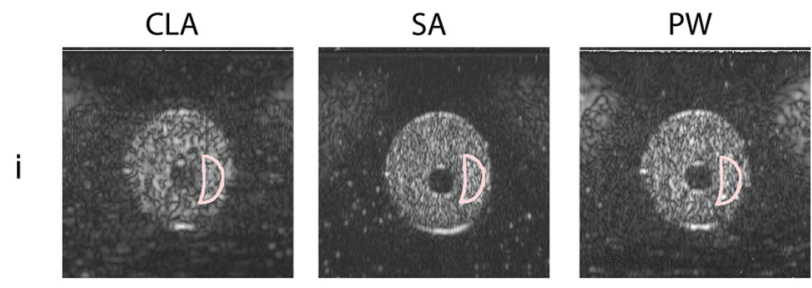

ii
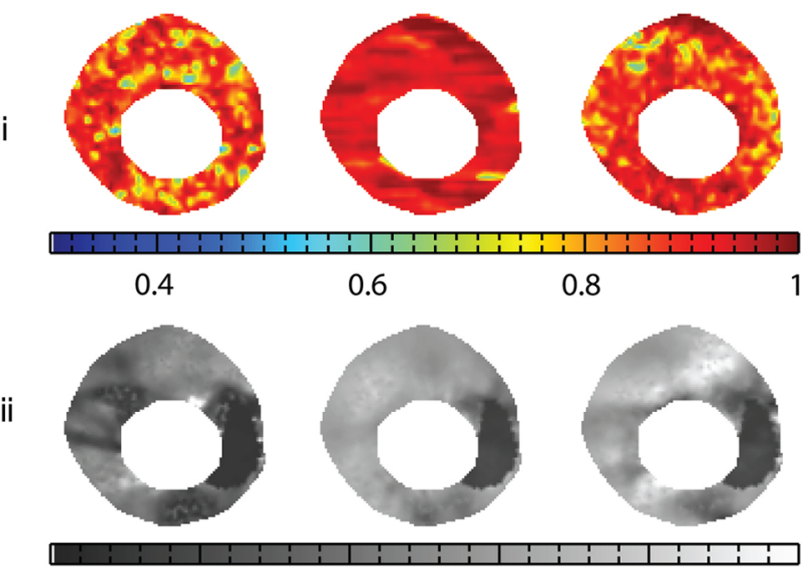

0 10
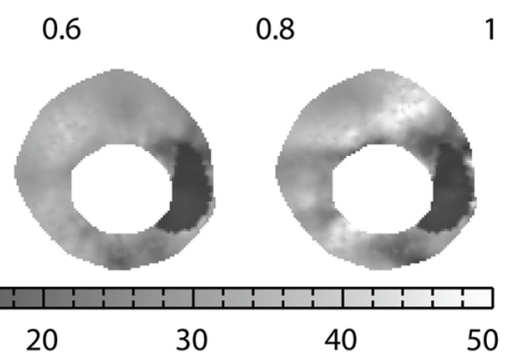

(a)

iv
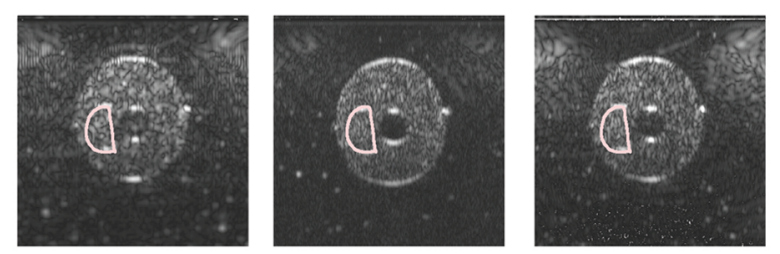

V
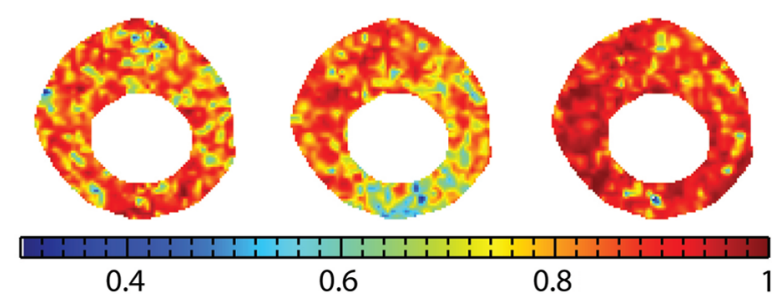

vi
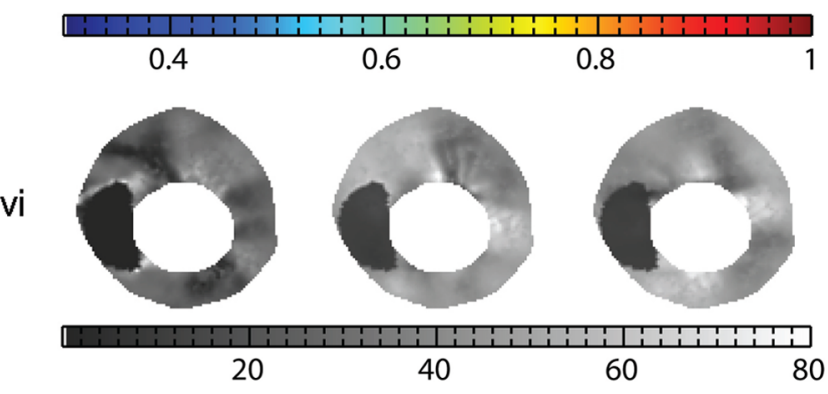

(b)

Fig. 9 Sonograms and elastograms obtained from vessel phantoms \#1 (a) and \#2 (b) when elastographic imaging was performed with CLA, SA, and PW imaging. (i and iv) Sonograms, (ii and v) cross-correlation maps, and (iii and vi) modulus elastogram. In the sonogram, the crescent shape region of interest $(\mathrm{ROI})$ denotes the plaque.

and 5-mm reception) was used to minimize the side-lobes. The larger apertures employed in SA and PW imaging (i.e., $38 \mathrm{~mm}$ for both transmission and reception) produced smaller lateral beam-widths of 0.18 and $0.23 \mathrm{~mm}$. Although similar size apertures were used in SA and PW, SA produced the tighter beam. This occurred because dynamic focusing was performed during
Table 2 Mean and standard deviation of Young's modulus of the plaques and vessel walls. These values were estimated from the recovered elastograms recovered with the three imaging approaches. We also report the values from independent mechanical testing of representative samples.

\begin{tabular}{lcccc} 
& MTS $(\mathrm{kPa})$ & $\mathrm{CLA}(\mathrm{kPa})$ & $\mathrm{SA}(\mathrm{kPa})$ & $\mathrm{PW}(\mathrm{kPa})$ \\
\hline $\begin{array}{l}\text { Phantom \#1: } \\
\text { plaque }\end{array}$ & $8.5 \pm 1.5$ & $6.1 \pm 1.8$ & $8.1 \pm 1.3$ & $7.4 \pm 1.3$ \\
$\begin{array}{l}\text { Phantom \#1: } \\
\text { vessel wall }\end{array}$ & $31.4 \pm 3.5$ & $29.2 \pm 3.9$ & $34.1 \pm 3.4$ & $30.7 \pm 2.1$ \\
$\begin{array}{l}\text { Phantom \#2: } \\
\text { plaque }\end{array}$ & $7.5 \pm 0.3$ & $4.2 \pm 0.9$ & $10.7 \pm 0.4$ & $7.2 \pm 0.1$ \\
$\begin{array}{l}\text { Phantom \#2: } \\
\text { vessel wall }\end{array}$ & $28.0 \pm 2.5$ & $21.7 \pm 8.1$ & $26.0 \pm 8.6$ & $32.1 \pm 8.4$ \\
\hline
\end{tabular}

transmission and reception in SA imaging, whereas in PW imaging this was done only during reception.

Strain elastograms acquired from the cross-sectional plane of the carotid arteries are difficult to interpret because strain is coordinate-dependent. Quantitative vascular elastography (QVE) can overcome this limitation by providing coordinate-independent mechanical parameters, as demonstrated in Figs. 3 and 4 . Von mises strain is also coordinate-invariant, ${ }^{46}$ but strain by itself does not provide any information about stress. In addition to improving the visual interpretation of the elastograms, QVE could (a) provide reliable modulus estimates that are required to compute stress, and (b) characterize the composition of different vessel components-information that could be used to identify life-threatening plaques and predict their propensity to rupture. However, successful translation of QVE to the clinic will depend on the availability of high-precision axial and lateral displacements-performing image reconstruction with limited displacement fields (i.e., only the axial component) produces less accurate modulus elastograms. $^{21}$

Figure 5 demonstrates the influence of noise on the performance (accuracy and precision) of modulus elastograms. In general, noise degrades the performance of inverse schemes and may even cause them to produce erroneous elastograms-a major challenge when solving ill-posed problems like ours. To circumvent this problem, smoothness constraints are used to stabilize the reconstruction process in the presence of noise. ${ }^{25,26}$ However, in vascular imaging, additional constraints (i.e., geometric) are required to produce stable modulus elastograms. ${ }^{32-35}$ Geometrically constrained inversion methods can tolerate higher noticeable amounts of measurement noise than those with just smoothness constraints. ${ }^{33,35}$ We performed a pair-wise Welch's $t$-test on the results reported in Fig. 7, which revealed that the modulus elastograms reconstructed with the three imaging methods were not significantly different $(p>0.05)$. We anticipated that this would occur because all reconstructions were performed with the optimum choice of the regularization parameter as determined using the L-curve method. ${ }^{35,43}$ However, in the phantom studies where it was difficult to chose the optimum values of regularization parameter, the results were different. More specifically, a pair-wise statistical analysis of the results (reported in Fig. 10) revealed no statistical differences $(p>0.05)$ in $\mathrm{PW}$ and SA modulus 


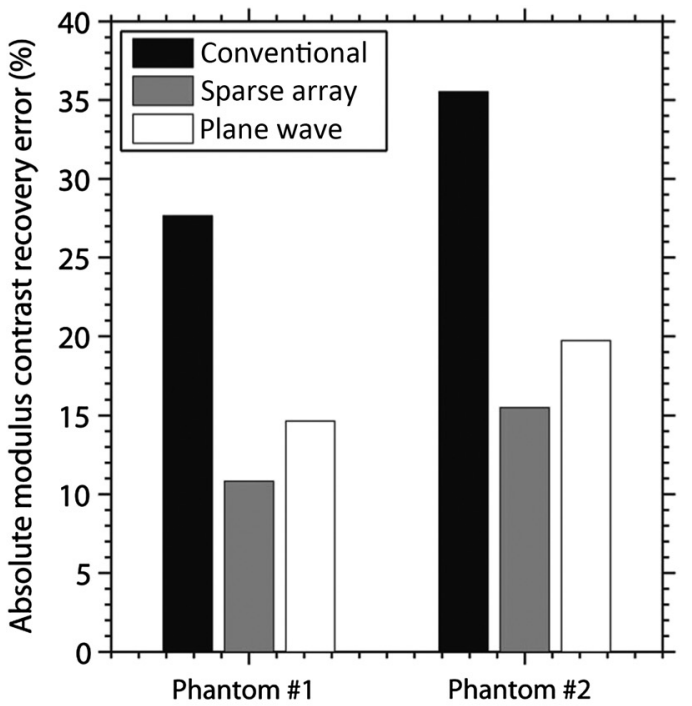

(a)

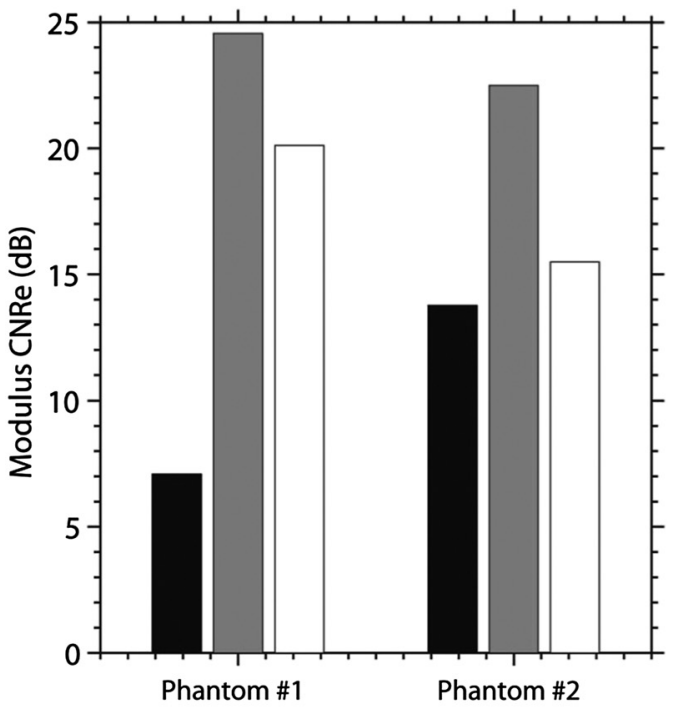

(b)

Fig. 10 (a) Contrast recovery error, and (b) contrast-to-noise ratio of modulus obtained with CLA, SA, $\mathrm{PW}$. Showing experimental results obtained from vessel phantoms with attenuation coefficients $0.6 \mathrm{~dB} /$ $\mathrm{cm}$ (phantom \# 1) and $3.5 \mathrm{~dB} / \mathrm{cm}$ (phantom \#2).

elastograms, but CLA modulus elastograms were significantly different $(p<0.05)$.

The main limitation of this study is that the dynamic range of moduli used in the vessel phantoms (simulated and physical) was low. More specifically, a typical vessel could have a $50-\mathrm{dB}$ modulus dynamic range between the fibrous cap $(100 \mathrm{kPa})$, fatty tissue $(16 \mathrm{kPa})$, and calcium deposits (5 MPa). There are concerns that local minima could prevent gradient-based reconstruction methods, such as those used in this study, from recovering such a wide range of modulus values. ${ }^{51,52}$ We are currently conducting studies to further investigate this issue in more detail. We plan to report the result of this investigation in a future communication.

\section{Conclusions}

In this work, we demonstrated that the qSAVE can produce accurate $(5 \%$ to $10 \%)$ modulus elastograms of superficial organs, such as the carotid artery. The logical next step of this research is to investigate whether modulus values recovered using qSAVE are accurate enough to compute useful stress elastograms of vascular tissues.

\section{References}

1. V. L. Roger et al., "Heart disease and stroke statistics-2012 update a report from the American heart association," Circulation 125(1), e2-e220 (2012).

2. J. Blacher et al., "Carotid arterial stiffness as a predictor of cardiovascular and all-cause mortality in end-stage renal disease," Hypertension 32(3), 570-574 (1998).

3. R. Virmani et al., "Pathology of the vulnerable plaque," J. Am. Coll. Cardiol. 47(8, Supplement), C13-C18 (2006).

4. Z. A. Fayad and V. Fuster, "Clinical imaging of the high-risk or vulnerable atherosclerotic plaque," Circ. Res. 89(4), 305-316 (2001).

5. K. Nieman et al., "Noninvasive angiographic evaluation of coronary stents with multi-slice spiral computed tomography," Herz 28(2), 136-142 (2003).

6. P. D. Richardson, M. J. Davies, and G. V. Born, "Influence of plaque configuration and stress distribution on fissuring of coronary atherosclerotic plaques," Lancet 334(8669), 941-944 (1989).
7. C. L. Lendon et al., "Atherosclerotic plaque caps are locally weakened when macrophages density is increased," Atherosclerosis 87(1), 87-90 (1991).

8. G. C. Cheng et al., "Distribution of circumferential stress in ruptured and stable atherosclerotic lesions. a structural analysis with histopathological correlation.," Circulation 87(4), 1179-1187 (1993).

9. C. Yuan et al., "Measurement of atherosclerotic carotid plaque size in vivo using high resolution magnetic resonance imaging," Circulation 98(24), 2666 (1998).

10. R. L. Maurice et al., "Adapting the lagrangian speckle model estimator for endovascular elastography: theory and validation with simulated radio-frequency data," J. Acoust. Soc. Am. 116, 1276-1186 (2004).

11. C. L. de Korte et al., "Identification of atherosclerotic plaque components with intravascular ultrasound elastography in vivo: a Yucatan pig study," Circulation 105(14), 1627-1630 (2002).

12. C. L. de Korte et al., "Characterization of plaque components and vulnerability with intravascular ultrasound elastography," Phys. Med. Biol. 45(6), 1465-1175 (2000).

13. J. A. Schaar et al., "Characterizing vulnerable plaque features with intra," Circulation 108, 2636-2641 (2003).

14. U. Techavipoo et al., "Estimation of displacement vectors and strain tensors in elastography using angular insonifications," IEEE Trans. Med. Imaging 23(12), 1479-1489 (2004).

15. M. Rao et al., "Normal and shear strain estimation using beam steering on linear-array transducers," Ultrasound in Med. Biol. 33(1), 57-66 (2007).

16. H. H. G. Hansen, R. G. P. Lopata, and C. L. de Korte, "Noninvasive carotid strain imaging using angular compounding at large beam steered angles: validation in vessel phantoms," IEEE Trans. Med. Imaging 28(6), 872-880 (2009).

17. H. H. G. Hansen et al., "An angular compounding technique using displacement projection for noninvasive ultrasound strain imaging of vessel cross-sections," Ultrasound Med. Biol. 36(11), 1947-1956 (2010).

18. H. H. G. Hansen et al., "Full 2D displacement vector and strain tensor estimation for superficial tissue using beam-steered ultrasound imaging," Phys. Med. Biol. 55(11), 3201-3218 (2010).

19. S. Korukonda et al., "Noninvasive vascular elastography using planewave and sparse-array imaging," IEEE Trans. Ultrason. Ferroelectr. Freq. Control 60(2), 332-342 (2013).

20. S. Korukonda and M. M. Doyley, "3D ultrafast elastography imaging of the carotid artery using sparse arrays," in 2011 IEEE Int. Ultrasonics Symp. (IUS), 2011 IEEE International, pp. 721-724, IEEE (2011). 
21. H. Hansen et al., "Noninvasive vascular displacement estimation for relative elastic modulus reconstruction in transversal imaging planes," Sensors 13, 3341-3357 (2013).

22. M. M. Doyley, "Model-based elastography: a survey of approaches to the inverse elasticity problem," Phys. Med. Biol. 57(3), R35 (2012).

23. M. Lubinski et al., "Lateral displacement estimation using tissue incompressibility," IEEE Trans. Ultrason. Ferroelectronics Freq. Control 43(2), 247-255 (1996).

24. S. Korukonda and M. Doyley, "Axial and lateral strain estimation using a synthetic aperture elastographic imaging system," Ultrasound Med. Biol. 37(11), 1893-1908 (2011).

25. M. M. Doyley, P. M. Meaney, and J. C. Bamber, "Evaluation of an iterative reconstruction method for quantitative elastography," Phys. Med. Biol. 45(6), 1521-1540 (2000).

26. F. Kallel and M. Bertrand, "Tissue elasticity reconstruction using linear perturbation method," IEEE Trans. Med. Imaging 15, 299-313 (1996).

27. A. A. Oberai et al., "Evaluation of the adjoint equation based algorithm for elasticity imaging," Phys. Med. Biol. 49(13), 2955-2974 (2004).

28. S. Timoshenko and J. Goodier, Theory of Elasticity, McGraw-Hill Classic Textbook Reissue Series, McGraw-Hill, York, PA (1969).

29. Y. Fung, Biomechanics, Mechanical Properties of Living Tissues, 2nd ed., Springer-Verlag, New York (1993).

30. P. E. Barbone and J. C. Bamber, "Quantitative elasticity imaging: what can and cannot be inferred from strain images," Phys. Med. Biol. 47(12), 2147-2164 (2002).

31. M. M. Doyley et al., "Enhancing the performance of model-based elastography by incorporating additional a priori information in the modulus image reconstruction process," Phys. Med. Biol. 51(1), 95 (2006).

32. R. A. Baldewsing et al., "Robustness of reconstructing the young's modulus distribution of vulnerable atherosclerotic plaques using a parametric plaque model," Ultrasound Med. Biol. 31(12), 1631-1645 (2005).

33. S. Le Floc'h et al., "Vulnerable atherosclerotic plaque elasticity reconstruction based on a segmentation-driven optimization procedure using strain measurements: theoretical framework," IEEE Trans. Med. Imaging 28(7), 1126-1137 (2009).

34. A. Samani, J. Bishop, and D. B. Plewes, "A constrained modulus reconstruction technique for breast cancer assessment," IEEE Trans. Med. Imaging 20(9), 877-885 (2001).

35. M. Richards and M. Doyley, "Investigating the impact of spatial priors on the performance of model-based ivus elastography," Phys. Med. Biol. 56(22), 7223-7246 (2011).

36. P. K. Yalavarthy et al., "Structural information within regularization matrices improves near infrared diffuse optical tomography," Opt. Express 15(13), 8043-8058 (2007).

37. E. E. Konofagou and J. Ophir, "A new elastographic method for estimation and imaging of lateral displacements, lateral strains, corrected axial strains and Poisson's ratio in tissues," Ultrasound Med. Biol. 24(8), 1183-1199 (1998).

38. R. Y. Chiao and L. J. Thomas, "Aperture formation on reduced-channel arrays using the transmit-receive apodization matrix," in IEEE Ultrasonics Symp., pp. 1567-1571, IEEE (1996).

39. S. Korukonda and M. M. Doyley, "Visualizing the radial and circumferential strain distribution within vessel phantoms using synthetic-aperture ultrasound elastography," IEEE Trans. Ultrason. Ferroelectr. Freq. Control 59(8), 1639-1653 (2012).

40. M. Bilgen and M. F. Insana, "Elastostatics of a spherical inclusion in homogeneous biological media," Phys. Med. Biol. 43(1), 1-20 (1998).
41. J. A. Jensen, "A model for the propagation and scattering of ultrasound in tissue," J. Acoust. Soc. Am. 89(1), 182-190 (1991).

42. R. Wagner et al., "Statistics of speckle in ultrasound b-scans," IEEE Trans. Sonics Ultrason. 30, 156-163 (1983).

43. C. R. Vogel, Computational Methods for Inverse Problems, Society for Industrial and Applied Mathematics, Philadelphia (2002).

44. S. Inglis et al., "An anthropomorphic tissue-mimicking phantom of the oesophagus for endoscopic ultrasound," Ultrasound Med. Biol. 32(2), 249-259 (2006).

45. J. Fromageau et al., "Estimation of polyvinyl alcohol cryogel mechanical properties with four ultrasound elastography methods and comparison with gold standard testings," IEEE Trans. Ultrason. Ferroelectr. Freq. Control 54, 498-509 (2007).

46. R. L. Maurice et al., "Non-invasive high-frequency vascular ultrasound elastography," Phys. Med. Biol. 50(7), 1611-1628 (2005).

47. K. J. M. Surry et al., "Poly(vinyl alcohol) cryogel phantoms for use in ultrasound and mr imaging," Phys. Med. Biol. 49(24), 5529 (2004).

48. M. M. Doyley et al., "Compartive evaluation of strain-based and modelbased modulus elastography," Ultrasound Med. Biol. 31(6), 787-802 (2005).

49. E. Konofagou and J. Ophir, "A new elastographic method for estimation and imaging of lateral displacements, lateral strains, corrected axial strains and Poisson's ratios in tissues," Ultrasound Med. Biol. 24(8), 1183-1199 (1998).

50. M. Karaman, P.-C. Li, and M. O'donnell, "Synthetic aperture imaging for small scale systems," IEEE Trans. Ultrason. Ferroelectr. Freq. Control 42, 429-442 (1995).

51. A. S. Khalil, B. E. Bouma, and M.R.K. Mofrad, "A combined FEM/ Genetic algorithm for vascular soft tissue elasticity estimation," Cardiovascular Eng. 6, 93-102 (2006).

52. A. S. Khalil et al., "Tissue elasticity estimation with optical coherence elastography: toward mechanical characterization of in vivo soft tissue," Ann. Biomed. Eng. 33(11), 1631-1639 (2005).

Steven Huntzicker received his bachelor's degree in general engineering from Harvey Mudd College in 2009. While there, he led multiple research projects in the area of algorithmic efficiency, both in software and hardware. Upon graduating, he joined the Electrical and Computer Engineering Department at the University of Rochester as a PhD student. His current research focuses on elastography, inverse problems, and cardiovascular imaging.

Rohit Nayak received his bachelor's degree in electrical and electronics engineering from Manipal University, India, in 2010. He completed his undergraduate thesis work at the Indian Institute of Science, working on surface plasmon resonance. He joined the Electrical and Computer Engineering Department at the University of Rochester as a PhD student in 2011. His research interests are in the areas of cardiovascular imaging, ultrasound beamforming, and elastography.

Marvin M. Doyley is an associate professor at the University of Rochester. He received his $\mathrm{PhD}$ degree in biophysics from the University of London in 2000. From 2000 to 2001, he was with the Thorax Center in The Netherlands. From 2001 to 2008, he was with the Thayer School of Engineering at Dartmouth College as a research faculty member. His research interests include cardiovascular imaging, molecular imaging, magnetic resonance elastography (MRE) and ultrasound elastography, and harmonic and modelbased imaging. 